\title{
Dimensionamiento y configuración teórica de una línea de cultivo de ostra perla (Pinctada imbricata Mollusca: Bivalvia), en la bahía de Charagato, isla de Cubagua, Venezuela*
}

\author{
Ernesto Trujillo ${ }^{1}$, Guillermo Martínez ${ }^{2}$ \& Luis León ${ }^{3}$ \\ ${ }^{1}$ Instituto de Investigaciones Científicas, Universidad de Oriente, Boca del Río \\ Apdo. 147, Porlamar, Estado de Nueva Esparta, Venezuela \\ ${ }^{2}$ Escuela de Ciencias del Mar, Pontificia Universidad Católica de Valparaíso \\ Casilla 1020, Valparaíso, Chile \\ ${ }^{3}$ Escuela de Ciencias Aplicadas del Mar (ECAM), Universidad de Oriente, Boca del Río \\ Apdo. 147, Porlamar, Estado de Nueva Esparta, Venezuela
}

\begin{abstract}
RESUMEN. Se dimensionó una línea de cultivo mediante análisis teórico de la resistencia hidrodinámica del sistema y se configuró utilizando simulación análoga con la ayuda de modelos a escala. Se determinaron las variables de tensión en línea madre (TLM), la relación de aspecto $\left(\mathrm{R}_{\mathrm{A}}\right)$ del fondeo y el excursionamiento $\left(\mathrm{P}_{\mathrm{R}}\right)$ de la línea madre, cuyas resultantes fueron la tensión en el sistema de fondeo $\left(\mathrm{T}_{2}\right)$ y el ángulo de ataque $\left(\alpha_{2}\right)$; la tensión vertical en una sección de línea madre (TF) y el ángulo de ataque de la catenaria $(\alpha)$. Para la construcción del modelo del cabo de fondeo y de una sección de línea madre se utilizaron factores de escala lineal de 40 y de 18,86; y escalas de fuerza de $9.776,3$ y de $1.233,13$, respectivamente. Las mínimas tensiones $\mathrm{T}_{2}$ fueron obtenidas para RA de $1 / 4$ y $1 / 5$, a partir de la tensión teórica $\mathrm{T}_{1}$ de 777,15 $\mathrm{kgf}$, con ángulos de ataque de $84,9^{\circ}$ y $90^{\circ}$. Para una sección de una línea de cultivo de seis linternas, con organismos en fase adulta, las unidades centrales 3 y 4 alcanzaron una profundidad operacional de 7,37 $\mathrm{m}$, una $\mathrm{P}_{\mathrm{R}}$ promedio de $93,6 \%$ y un ángulo de ataque de $55,86^{\circ}$. Se requiere validar in situ los resultados teóricos obtenidos, con ayuda de un tensiómetro, a fin de encontrar el equilibrio estructural del sistema con un mínimo de inversión y máximo rendimiento.
\end{abstract}

Palabras clave: dimensionamiento, tensión, sistema de fondeo, línea de cultivo, simulación análoga, acuicultura, ostra perla.

\section{Sizing and theoretical configuration of a longline for growing pearl oysters (Pinctada imbricata Mollusca: Bivalvia) in the Bay of Charagato, Cubagua Island, Venezuela*}

\begin{abstract}
A farming longline was sized by theoretically analyzing the system's hydrodynamic resistance and configured using analogue simulation with the help of scale models. The variables determined were mother line tension (TLM), the relationship of the anchoring aspect $\left(\mathrm{R}_{\mathrm{A}}\right)$, and movement $\left(\mathrm{P}_{\mathrm{R}}\right)$ of the mother line. These resulted in anchoring system tension $\left(\mathrm{T}_{2}\right)$ and attack angle $\left(\alpha_{2}\right)$, and the vertical tension for a section of the mother line (TF) and the attack angle of the catenary $(\alpha)$. The model of the anchor end and a section of the mother line were constructed using lineal scale factors of 40 and 18.86 and force scales of $9,776.3$ and 1,233.13, respectively. The minimum tensions $\left(T_{2}\right)$ obtained for $R_{A}$ were $1 / 4$ and $1 / 5$ based on the theoretical tension $\left(T_{1}\right) 777.15 \mathrm{kgf}$, with attack angles of $84.9^{\circ}$ and $90^{\circ}$. A longline section with six lanterns and loaded with adult organisms had central units 3 and 4 at an operational depth of $7.37 \mathrm{~m}$, an average $\mathrm{P}_{\mathrm{R}}$ of $93.6 \%$, and an attack angle of $55.86^{\circ}$. A tensiometer should be used for an in situ validation of the theoretical results in order to determine the structural equilibrium of the system with minimum investment and maximum performance.
\end{abstract}

Key words: sizing, tension, anchoring system, farming longline, analogue simulation, aquaculture, pearl oyster.

Autor corresponsal: Guillermo Martínez (guillermo.martinez@ucv.cl).

* Trabajo presentado en el XXV Congreso de Ciencias del Mar de Chile y XI Congreso Latinoamericano de Ciencias del Mar (COLACMAR), realizados en Viña del Mar, entre el 16 y 20 de mayo de 2005. 


\section{INTRODUCCIÓN}

Existe un interés particular en el cultivo de la ostra perla (Pinctada imbricata), principalmente por sus características de rápido crecimiento y alta supervivencia en condiciones de cultivo suspendido, además de la disponibilidad de semillas a través del año. No obstante, en Venezuela la maricultura es una actividad reciente que no ha experimentado un gran desarrollo con especies nativas, debido a que la investigación no se ha realizado en forma planificada y progresiva, siendo más bien casuística y empírica (Lodeiros, 1999).

Dentro de la factibilidad de cultivo de especies de bivalvos, reflejada en la disponibilidad de semilla o juveniles y en crecimiento, también se agrega la adaptabilidad de las especies a su medio ambiente. Por tal razón se hace necesario el estudio de sensibilidad de los organismos bajo cultivo a los cambios ambientales, dado a que el nororiente del país se encuentra influenciado por la surgencia que genera una variabilidad hidrobiológica, normalmente de forma estacional, que conduce a la modulación de procesos fisiológicos de muchos invertebrados acuáticos de dicha región (Lodeiros \& Himmelman, 1994; Lodeiros, 1996).

En cuanto a la existencia de una metodología para el dimensionamiento y modelaje de sistemas de líneas, se puede decir que sólo se ha hecho uso de metodologías específicas para diseño de artes de pesca, diseño de sistemas de fondeo de balizas oceanográficas y plataformas petroleras, entre otras, para adaptarlas a las necesidades de dimensionamiento de sistemas de líneas de cultivo. Por ello, se ha tenido que establecer un marco referencial a través del análisis cualitativo de cada una de ellas (Martínez \& Carroza, 1992).

Entre estas metodologías se encuentra la propuesta por Berteaux (1976) quien realizó estudios sobre el comportamiento de sistemas de fondeo de boyas oceanográficas, estableciendo como premisas que el cable no sufre elongación bajo tensión, la dirección de la corriente no tiene componente vertical y la magnitud de la corriente es constante en toda la columna de agua. Para el estudio del comportamiento de las líneas de fondeo, este autor consideró necesario conocer previamente las fuerzas gravitacionales, la resistencia al arrastre del órgano objeto de estudio y la tensión en la línea por efecto de la resistencia aerodinámica de los cuerpos flotantes.

Asimismo, Neshyba \& Fonseca (1981) analizaron los diversos aspectos técnicos de los fondeos para las boyas oceanográficas y establecieron que todo cuerpo sumergido experimenta una resistencia al arrastre ante el movimiento del flujo de agua. También establecieron que las olas ejercen un efecto adicional de tensión sobre un cuerpo que se encuentra anclado en el fondo, siendo la máxima igual a la suma de la tensión estática y la tensión dinámica, como resultado de las fuerzas horizontales y de las olas, respectivamente.

Miyazaki (1970) planteó un procedimiento de cálculo teórico mediante el estudio de la forma y transmisión de tensión sobre un cabo, el cual adopta la forma de catenaria, y sus resultados fueron validados con los de tipo experimental, para lo cual estableció un margen de error de $( \pm)$ 10\%.

Carroza (1990) trabajó sobre aspectos metodológicos para la selección y dimensionamiento del sistema de anclaje de una línea de cultivo adaptando los estudios de Berteaux (1976), Neshyba \& Fonseca (1981) y Miyazaki (1970), entre otros.

Martínez et al. (1990), hicieron estudios sobre el dimensionamiento teórico y selección de componentes para una línea de cultivo de ostión del norte (Argopecten purpuratus), apoyándose en metodologías específicas. Para ello, incorporaron fórmulas diseñadas para otras estructuras, tales como artes de pesca, almadrabas, boyas, orinques de fondeo, etc.; para operar en ambientes marinos sometidas a fuerzas hidrodinámicas generadas por las corrientes o mareas existentes en el medio, de la misma forma como son sometidas las líneas de cultivo en condiciones de trabajo.

Para la configuración del sistema de línea de cultivo propuesto, se utiliza el método de Analogía Mecánica desarrollado por Baranov $(1939 ; 1966)$ para el diseño de artes de pesca industriales, cuyo enfoque estuvo dirigido hacia sistemas de hilo flexible. El mismo se fundamenta en la preparación y experimentación de un modelo análogo del esquema de cálculo de un arte de pesca o de sus partes, donde las líneas geométricas en el esquema se reemplazan por hilos flexibles, a los cuales se aplican fuerzas externas en sus respectivas secciones (Martínez et al., 1990). Este método ha sido profundizado y detallado por Andreyev (1962), Kondratyev (1973), VoinikanisMirskii (1979) y Fridman (1981,1986), entre otros. A tal efecto, Voinikanis-Mirskii (1966) recomienda el uso de métodos simplificados de modelación, la modelación estática, donde los procesos físicos se imitan en condiciones estáticas.

La configuración de catenaria que adoptan las 
secciones de una línea de cultivo, como la línea madre y cabos de fondeo, depende directamente de las características de los materiales empleados en cada una de las partes y del diseño empleado para su construcción, debido a que ellos se encuentran bajo la acción de pesos proporcionales a su longitud (fuerza de gravedad).

También influyen sobre la forma de la catenaria los factores físicos, tales como dirección y velocidad de la corriente en la columna de agua y en la superficie, dirección y velocidad del viento; además de factores operativos, como la posición de la línea de cultivo con relación a la corriente y al viento. Del mismo modo, el peso de las unidades de cultivo también influye en la forma que adopta la línea madre y sistemas de fondeo $y$, por ende, en la configuración de la línea de cultivo.

Martínez \& Carroza (1992) utilizaron la técnica de simulación análoga para determinar la configuración de un cabo de fondeo de una línea de cultivo, a objeto de minimizar la tensión resultante sobre el sistema de anclaje. Esta misma técnica fue utilizada por Trujillo \& Martínez (2003) para estudiar la forma y tensión que se produce en una sección de línea madre de un palangre atunero, utilizando diferentes proporciones de recorte. Por otro lado, Raman-Nair $\&$ Colbourne (2003) estudiaron el comportamiento de un sistema de línea de cultivo de mejillones en condiciones sumergidas, mediante la técnica de modelamiento numérico.

En cuanto a la experiencia en Venezuela, sobre desarrollo de cultivos y aspectos bioecológicos en moluscos bivalvos, se encuentran algunos trabajos realizados con especies de mejillón (Perna perna) (León, et al., 1998), ostra perla (Pinctada imbricata) (León, et al., 1987; León \& Pereyra, 1994; León \& Millán, 1996; León, 1997); de ostra de mangle (Crassostrea rizophorae) (Díaz et al., 1998), entre otros; además de estudios hidrográficos y de fitoplancton en la isla de Cubagua (Troccoli \& Cabrera, 1994a, 1994b).

Por otra parte, Lodeiros et al. (2002) estudiaron el crecimiento y sobrevivencia de la ostra perla (Pinctada imbricata) en cultivos suspendidos y de fondo en el golfo de Cariaco. En tanto que Lodeiros \& García (2004), estudiaron el uso de erizos de mar para el control de incrustantes durante el cultivo de bivalvos suspendidos.

No obstante, sobre diseño y dimensionamiento estructural de sistemas flexibles para el cultivo de moluscos bivalvos, desde el punto de vista de la ingeniería, no se tienen antecedentes en Venezuela. Por esta razón, la presente investigación tiene como principal objetivo dimensionar y configurar teóricamente un sistema de línea de cultivo de ostra perla (Pinctada imbricata), en la bahía de Charagato, isla de Cubagua, Venezuela.

\section{MATERIALES Y MÉTODOS}

Para la definición de los componentes del sistema de cultivo tipo línea de cultivo (longline) se utilizó un esquema, cuya secuencia metodológica fue adaptada de las propuestas realizadas por Carroza (1990) y Martínez \& Carroza (1992). Mientras que, para el dimensionamiento del sistema de cultivo, en general, se analizaron y determinaron cada uno de sus componentes mediante metodologías específicas, de acuerdo a las sugerencias de Martínez et al. (1990) y Martínez \& Carroza (1992). A tal efecto, con un enfoque sistémico, se realizó un estudio del comportamiento de cada uno de los componentes de la línea de cultivo en relación con su entorno.

Para determinar la forma y tensión transmitida al cabo de fondeo, así como la forma y tensión en la línea madre, se utilizó la simulación mecánica o análoga descrita por Martínez \& Carroza (1992) y Trujillo \& Martínez (2003), respectivamente. Ambos ensayos se realizaron en forma independiente, tomando como dato de entrada los resultados obtenidos previamente, del cálculo teórico de la resistencia hidrodinámica del sistema.

El diseño de las unidades de cultivo se realizó mediante un análisis teórico de estos sistemas, considerando las características de forma, comportamiento hidrodinámico, material, tamaño y componentes de las unidades, de acuerdo con el propósito del sistema y las restricciones del diseño.

La forma de la unidad de cultivo, para la especie de ostra perla, así como el material y sus componentes, fueron seleccionados entre los diversos sistemas de uso en el mercado internacional. Se seleccionó la linterna, en virtud a su eficiencia comprobada en experiencias destinadas al cultivo suspendido de moluscos bivalvos, como lo reportan los trabajos de Carroza (1990) y Martínez \& Carroza (1992).

Por cuanto la línea de cultivo se encuentra afectada por un conjunto de fuerzas externas que determinan su forma y tensiones, a las cuales está sometida. El estudio del comportamiento hidrodinámico de cada uno de los componentes del sistema 
y del conjunto en general se realizó considerando la resistencia que el cuerpo opone al medio en posición de trabajo, perpendicular al flujo, utilizando la ecuación de Fridman (1981) para la hidromecánica aplicada:

$$
\mathrm{R}_{\text {Sistema }}=\mathrm{C}_{\mathrm{x}} \cdot \rho \cdot \mathrm{v}^{2} \cdot \mathrm{F} / 2
$$

donde:

$$
\begin{aligned}
& \mathrm{R}_{\text {Sistema }} \text { : resistencia hidrodinámica del sistema } \\
& \text { objeto, kgf } \\
& \rho \quad: \text { densidad del fluido, } \mathrm{kg} \cdot \mathrm{m}^{-3} \\
& \mathrm{C}_{\mathrm{x}} \quad \text { : coeficiente de resistencia hidrodinámica }
\end{aligned}
$$

En esta expresión, el coeficiente de resistencia $\left(C_{x}\right)$ se determina de acuerdo al Número de Reynolds (Re):

$$
\mathrm{R}_{\mathrm{e}}=1 \mathrm{v} / \mathrm{v}
$$

donde:

$$
\begin{array}{ll}
1 & : \begin{array}{l}
\text { longitud característica del cuerpo (diáme- } \\
\text { tro para los cilindros } \mathrm{y} \text { cabos), } \mathrm{m}
\end{array} \\
\mathrm{v} & : \text { velocidad del flujo, } \mathrm{m} \cdot \mathrm{s}^{-1} \\
\mathrm{v} & : \text { viscosidad cinemática del fluido, } \mathrm{m}^{2} \cdot \mathrm{s}^{-1}
\end{array}
$$

Para determinar el coeficiente de resistencia $\left(\mathrm{C}_{\mathrm{x}}\right)$ se siguió el criterio recomendado por Martínez et al. (1990):

$$
\mathrm{C}_{\mathrm{x}}=\mathrm{a} \cdot\left(\mathrm{R}_{\mathrm{e}}\right)^{\mathrm{b}}
$$

Estos autores establecieron que para $R_{e}>1000$ $\mathrm{a}=1,28$ y $\mathrm{b}=0,011$; mientras que para $\mathrm{R}_{\mathrm{e}}<1000 \mathrm{a}$ $=8,5 \mathrm{y} \mathrm{b}=-0,85$.

\section{Dimensionamiento y selección de cabos estructurales}

Para el dimensionamiento de los cabos estructurales se determinó la resistencia a la ruptura $\left(\mathrm{R}_{\text {rup }}\right)$ de acuerdo al material de construcción, con un factor de seguridad para compensar las fluctuaciones de tensión que se producen en las líneas por razones ambientales, conforme a las recomendaciones de Berteaux (1976). En tal sentido, se seleccionaron los materiales de acuerdo a las propiedades técnicas, tipo de construcción, peso específico, capacidad de absorción en el agua, abrasión, aspectos operativos en condiciones sumergidas y otras propiedades de interés para la construcción del sistema, conforme a las recomendaciones dadas por Martínez et al. (1990).

Para determinar la longitud de la línea madre se consideraron factores tales como área de trabajo disponible en la isla de Cubagua, recursos económicos para la inversión inicial del proyecto, compra de material de cabo de polietileno (PE) disponible en el mercado local y productividad del medio ambiente. Esta última, determina la capacidad de carga que permite establecer una densidad óptima de cultivo de la especie seleccionada. Por ello, se hizo un estudio de línea base ambiental consistente en el muestreo del área durante un año, donde tuvo gran relevancia la determinación de la concentración de nutrientes en el medio sujeto a variaciones ambientales, tales como surgencia, fenómenos oceanográficos, oxígeno disuelto, etc., así como la conveniente separación entre las unidades de cultivo, lo cual influye en la capacidad de carga del sistema de cultivo.

El manejo de la línea madre y su resistencia hidrodinámica fueron otros de los factores considerados en el dimensionamiento del sistema, en virtud de que las unidades de cultivo influyen sobre dichos factores. También se realizó el cálculo del diámetro teórico de la línea madre, el cual se fundamentó en datos de entrada obtenidos de otras experiencias con estos tipos de estructuras.

Las características de los cabos de fondeo se determinaron de acuerdo a consideraciones tales como: longitud, que se establece en función de la profundidad de la zona de instalación elegida para el sistema; relación de aspecto aplicada, es decir, relación profundidad/longitud del cabo de fondeo; y diámetro, que se determina de acuerdo a las tensiones, tanto de referencia como experimentales (simuladas), para asegurar que no se produzca una ruptura durante la operación.

Las características de los cabos de flotadores superficiales se determinaron sobre la base de profundidad de la línea madre, profundidad del lugar de instalación y diámetro, el cual se determina mediante el cálculo de la tensión en condiciones de trabajo. Para ello se requirió calcular el peso sumergido que debe soportar el sistema de flotación de la línea de cultivo. 


\section{Dimensionamiento y selección del sistema de flotación}

El sistema de flotación tiene como función sustentar verticalmente la línea madre en la columna de agua, en la profundidad de trabajo establecida, lo cual se logra gracias al sistema de flotación superficial y sistema de flotación de media agua.

La selección de la flotación apropiada se determinó en función de los requerimientos de crecimiento y sobrevivencia de la especie, que hacen que la unidad de cultivo permanezca sumergida durante todo el proceso; de las características ambientales del medio, tales como profundidad, mareas, corrientes y existencia de olas y, por último, de la disponibilidad de flotadores en desuso en el mercado, lo cual condicionaba el costo del proyecto.

La flotabilidad de un flotador se calculó tomando como referencia la tensión vertical $\left(\mathrm{T}_{\mathrm{y}}\right)$, considerando la profundidad de trabajo del flotador y un margen de seguridad apropiado. Si se asume que este valor es del $20 \%$ de la flotabilidad de cada flotador, la flotabilidad requerida por el flotador se calculó por medio de la siguiente ecuación:

$$
\mathrm{Q}_{\mathrm{f}}=1,20 \mathrm{~T}_{\mathrm{y}}
$$

donde:

$\mathrm{Q}_{\mathrm{f}} \quad$ : flotabilidad necesaria para el flotador, kgf

Esto significa que cada flotador debe desarrollar esta fuerza $\left(Q_{f}\right)$ para poder compensar el peso lineal encontrado, que se genera a lo largo de la línea madre.

Los flotadores de media agua son de PVC expandido, denso, que se utilizan en las redes de cerco atunero, cuya flotabilidad se calculó teóricamente, tomando en cuenta su volumen y su densidad:

$$
\begin{gathered}
\mathrm{V}_{\mathrm{f}}=\pi / 4 \mathrm{~L}_{\mathrm{f}}\left(\mathrm{D}^{2}-\mathrm{DI}^{2}\right) \\
\mathrm{Q}_{\mathrm{f}}=\left(\rho_{\mathrm{A}} \mathrm{V}_{\mathrm{f}}\right)-\mathrm{P}_{\mathrm{fA}}
\end{gathered}
$$

donde:

$$
\begin{array}{ll}
\mathrm{V}_{\mathrm{f}} & \text { : volumen del flotador, } \mathrm{cm}^{3} \\
\mathrm{~L}_{\mathrm{f}} & : \text { longitud del flotador, } \mathrm{cm} \\
\mathrm{D} & \text { : ancho del flotador, } \mathrm{cm} \\
\mathrm{DI} & : \text { diámetro interior del agujero (buje), } \mathrm{cm} \\
\rho_{\mathrm{A}} & : \text { densidad del agua, } \mathrm{kg} \cdot \mathrm{m}^{-3}
\end{array}
$$

Para corregir la flotabilidad teórica del flotador $\left(\mathrm{Q}_{\mathrm{fr}}\right)$, se utilizó un factor de pérdida entre el 25 y
$30 \%$, por ser material en desuso que provoca pérdida de su flotabilidad original por efecto del deterioro sufrido. Por lo tanto:

$$
\mathrm{Q}_{\mathrm{fr}}=\mathrm{Q}_{\mathrm{f}}(0,25 \sim 0,30)
$$

\section{Cálculo del peso total de la línea de cultivo}

El peso total del sistema de la línea de cultivo en condiciones operativas, resultó de la sumatoria de todos los pesos sumergidos que constituyen el sistema, que se determinó de acuerdo a las siguientes expresiones (Voinikanis-Mirskii, 1971):

$$
\begin{gathered}
\mathrm{P}_{\text {Total }}=\sum \mathrm{Q}_{1 \mathrm{i}} \\
\mathrm{Q}_{1 \mathrm{i}}=\mathrm{G}_{\mathrm{i}}\left(1-\rho_{\mathrm{A}} / \rho_{\mathrm{Mi}}\right)
\end{gathered}
$$

donde:

$\mathrm{P}_{\text {Total }}$ : peso total sumergido de la línea de cultivo, $\mathrm{kg}$

$\mathrm{Q}_{1 \mathrm{i}} \quad$ : peso sumergido del componente $\mathrm{i}, \mathrm{kg}$

$\mathrm{G}_{\mathrm{i}} \quad$ : peso en el aire del componente $\mathrm{i}, \mathrm{kg}$

$\rho_{\mathrm{Mi}}:$ densidad del material del componente $\mathrm{i}$, $\mathrm{kg} \cdot \mathrm{m}^{-3}$

$\rho_{\mathrm{A}} \quad:$ densidad del agua de $\mathrm{mar}, \mathrm{kg} \cdot \mathrm{m}^{-3}$

\section{Cálculo del peso lineal de la línea de cultivo}

Determinado el peso sumergido total de la línea de cultivo, se calculó el peso por unidad de longitud de la línea madre $\left(\mathrm{F}_{\mathrm{S}}\right)$ de acuerdo a las recomendaciones de Fridman (1986), por cuanto esta estructura es la que soporta las tensiones ejercidas por la acción de los flotadores. Este peso se determinó mediante la siguiente ecuación:

$$
\mathrm{F}_{\mathrm{S}}=\mathrm{P}_{\text {Total }} / \mathrm{L}_{\mathrm{LM}}
$$

donde:

$\mathrm{F}_{\mathrm{S}} \quad$ : peso por unidad de longitud, $\mathrm{kg} \cdot \mathrm{m}^{-1}$

$\mathrm{L}_{\mathrm{LM}} \quad$ : longitud de la línea madre, $\mathrm{m}$

\section{Dimensionamiento y selección del sistema de anclaje}

Para el dimensionamiento y selección del sistema de anclaje se consideraron factores tales como condiciones del fondo (tipo de sustrato), disponibilidad del sistema de anclaje en el mercado y características de la tensión transmitida al sistema de fondeo. 
En este caso, se supuso que el cabo de fondeo adopta una configuración de catenaria, la cual indica que la tensión que se produce, en la unión de este cabo con la línea madre, es transmitida en su totalidad al sistema de anclaje. Por ello, la tensión crítica que se produce en el cabo de fondeo equivale a la suma de la tensión máxima calculada, más la tensión en el cabo de fondeo producida por la resistencia hidrodinámica del sistema, sometida a una corriente igual a $0,47 \mathrm{~m} \cdot \mathrm{s}^{-1}$. Esta última fue monitoreada in situ durante un año y su cálculo fue el resultado del promedio de todas las muestras. En tal sentido, al haber determinado la resistencia (tensión) en forma teórica, a la cual se somete la línea de cultivo, conforme a la ecuación de Fridman (1981) (1), se procedió al cálculo de la resistencia al flujo del cabo de fondeo $\left(\mathrm{R}_{\mathrm{cf}}\right)$, su peso en el agua (w) y el ángulo de ataque crítico del cabo $\Phi_{\mathrm{c}}$.

Conociendo los valores de $\mathrm{Q}_{\mathrm{f} 1}$ (flotabilidad del primer flotador-baliza), $\mathrm{P}_{\mathrm{cl}}$ (peso de la primera linterna) y $\mathrm{T}_{1}$ (tensión de la línea madre en el Punto 1 ), se calculó el vector de tensión transmitida en el Punto $1\left(\mathrm{t}_{1}\right)$, así como el ángulo de ataque $\left(\Phi_{1}\right)$ del vector $\mathrm{t}_{1}$, y la tensión en el Punto $2\left(\mathrm{t}_{2}\right)$, que corresponde al punto de unión del cabo de fondeo con el anclaje.

\section{Elementos de anclaje propuesto}

Para la línea de cultivo prototipo "Margarita" se seleccionó un ancla tipo rezón tradicional de cinco uñas, para ser utilizado en un sustrato de fondo areno-fangoso y una relación de aspecto tentativa $\left(\mathrm{R}_{\mathrm{A}}\right)$ de $1 / 3$, entre la profundidad de la línea madre y la longitud del cabo de fondeo.

$\mathrm{Al}$ no conocer el coeficiente de agarre $(\mathrm{K})$ del ancla seleccionada, se utilizó un coeficiente de 17,5 recomendado por Ogg (1969), para anclas tipo $L W T$ (light weight) en fondos fangosos, en virtud a que éstas se asemejan en su dinámica al típico rezón de cinco uñas. Por lo tanto, el poder de agarre $\left(\mathrm{P}_{\mathrm{a}}\right)$, de acuerdo a las características del fondo y al ángulo de ataque formado entre el cabo de fondeo y el fondo marino, estará dado por:

$$
\text { Poder de agarre }\left(\mathrm{P}_{\mathrm{a}}\right)=\mathrm{K} \cdot \mathrm{W}
$$

donde:

W : peso sumergido del ancla, $\mathrm{kg}$

\section{Determinación de los accesorios del sistema}

Los accesorios tienen como finalidad conectar los distintos componentes del sistema de la línea de cultivo, entre ellos se encuentran los grilletes, guardacabos, grilletes-giratorios y argollas. Su selección se realizó de acuerdo a los requerimientos del sistema, a las características de los cabos y tensiones encontradas en cada uno de los puntos de unión. La determinación se efectuó de acuerdo a lo recomendado por Martínez et al. (1990) y se emplearon catálogos disponibles en el mercado para seleccionarlos acorde a sus características y dimensiones requeridas.

\section{Configuración del cabo de fondeo y de una sección de línea madre}

La configuración del cabo de fondeo y de una sección de línea madre de la línea de cultivo se realizó tomando como dato de entrada el cálculo teórico de la tensión en la línea madre y en el cabo de fondeo. Para ello se utilizó el método de simulación análoga, de acuerdo a Baranov (1969), Kondratyev (1973), Fridman (1981), Carroza (1990), Martínez \& Carroza (1992) y Trujillo \& Martínez (2003).

Para realizar la simulación, el perfil de la sección del cabo de fondeo y de una sección de línea madre se reemplazaron por un hilo flexible, con su correspondiente escala lineal de similitud $\left(\mathrm{C}_{\mathrm{L}}\right)$. De igual forma, las fuerzas externas se imitaron en forma de pesos, sustentadas en su respectiva escala de fuerzas $\left(\mathrm{C}_{\mathrm{R}}\right)$. Las constantes geométricas y de fuerzas se consideraron independientes entre sí, por lo que el modelo se ejecutó de una forma, y las fuerzas se ejecutaron, independientemente, en otra escala. En tal sentido, las escalas se determinaron de acuerdo a lo recomendado por Baranov (1969), Kondratyev (1973) y Fridman (1981).

\section{Criterios para la simulación}

Para la simulación se tomó como marco de referencia los trabajos realizados por Martínez \& Carroza (1992) y Trujillo \& Martínez (2003) que posibilitaron determinar, por una parte, la tensión en los puntos de unión entre el cabo del primer flotador, la línea madre y el cabo de fondeo con anclaje y, por otra parte, la forma y tensión de una sección de línea madre sustentada entre dos balizas, con sus respetivas unidades de cultivo.

Para la simulación en laboratorio de los componentes de semejanza de fuerza (peso) y geométrica (forma) a escala, se definió la siguiente secuencia:

Escala lineal $\left(\mathrm{E}_{\mathrm{L}}\right)$ : Se seleccionó de acuerdo al tamaño del panel de prueba y a las facilidades para trabajar sobre él. Por lo tanto, se tomó como escala 
lineal la relación entre la longitud del prototipo $\left(\mathrm{L}_{\mathrm{N}}\right)$ y la longitud del modelo $\left(\mathrm{L}_{\mathrm{M}}\right)$

$$
\mathrm{L}_{\mathrm{M}}=\mathrm{L}_{\mathrm{N}} / \mathrm{E}_{\mathrm{L}}
$$

Escala de fuerza $\left(\mathrm{E}_{\mathrm{F}}\right)$ : Para el escalamiento de los pesos se tomó la relación entre las fuerzas que actúan sobre la estructura al natural $\left(\mathrm{F}_{\mathrm{N}}\right)$ y las fuerzas que actúan sobre el modelo $\left(\mathrm{F}_{\mathrm{M}}\right)$ :

$$
\mathrm{F}_{\mathrm{M}}=\mathrm{F}_{\mathrm{N}} / \mathrm{E}_{\mathrm{F}}
$$

\section{Modelamiento en laboratorio}

En laboratorio se simuló un cabo de fondeo y una sección de línea madre con seis unidades de cultivo, utilizando como datos de entrada las condiciones oceanográficas obtenidas in situ, y los resultados del cálculo teórico de la resistencia total del sistema. A tal efecto, se utilizó el procedimiento de simulación análoga adoptado por Martínez \& Carroza (1992) y Trujillo \& Martínez (2003), para la construcción del modelo (Figs. 1 y 2 ).

\section{Experimento y análisis de datos}

La finalidad de estos experimentos fue, por una parte, establecer la configuración de diseño del cabo de fondeo en función de la relación de aspecto del mismo,

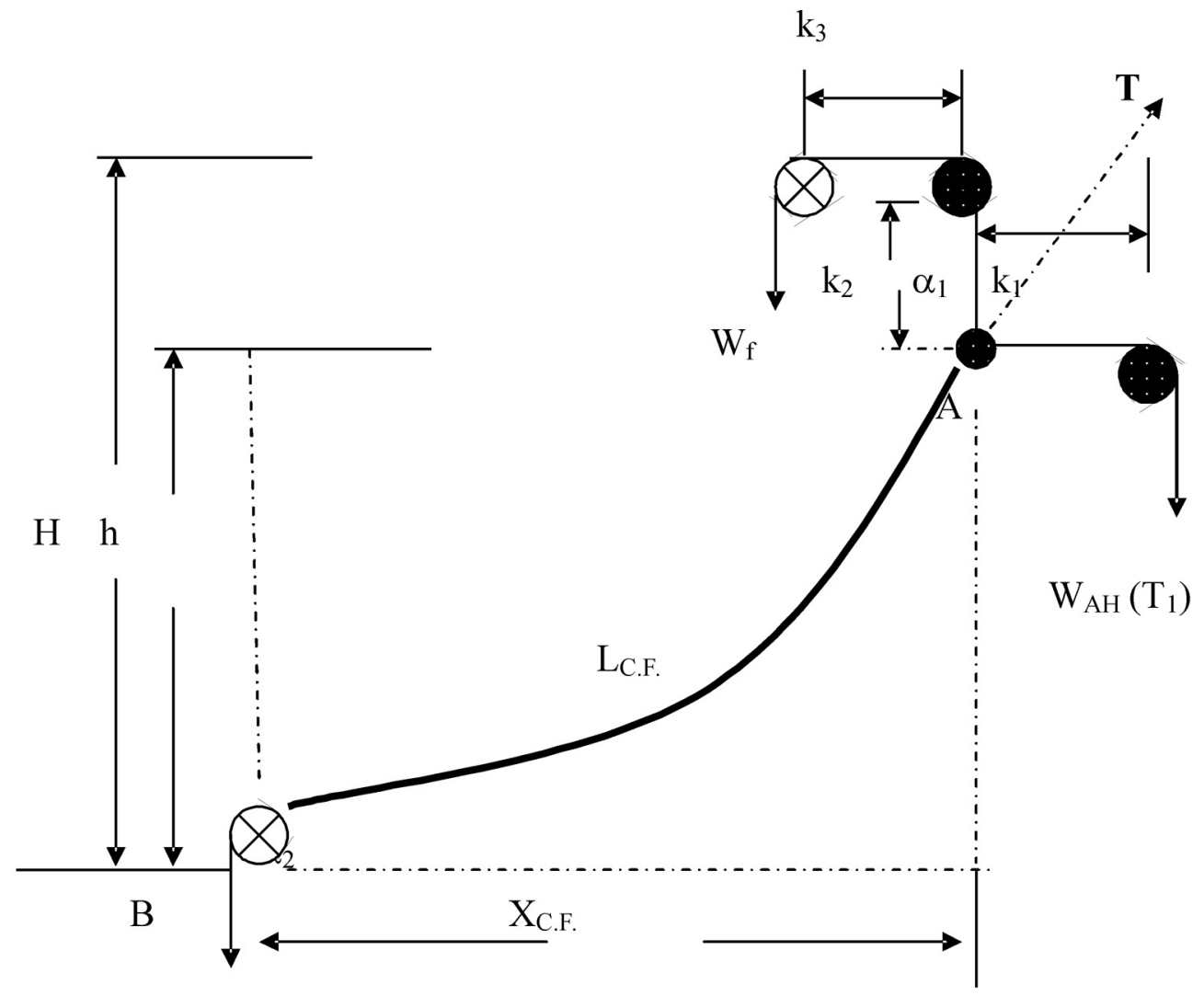

$$
\mathrm{W}_{\text {B.H. }}\left(\mathrm{T}_{2}\right)
$$

Figura 1. Esquema del modelo utilizado en la simulación mecánica del cabo de fondeo (adaptado de Martínez \& Carroza, 1992). $W_{A H}$ : tensión de prueba en el punto $A\left(T_{1}\right), W_{f}$ : tensión del primer flotador, $L_{C . F}$ : longitud del cabo de fondeo, $H$ : profundidad total, $h$ : profundidad de la línea madre respecto al fondo, $W_{B . H}$ : tensión resultante en el punto $B\left(T_{2}\right), X_{C . F}$ : excursión del fondeo $(I=1-6), \alpha_{1}, \alpha_{2}$ : ángulos de ataque teórico de línea madre y fondeo, $\mathbf{k}_{1}, \mathbf{k}_{2}, \mathbf{k}_{3}$ : distancias constantes.

Figure 1. Scheme of the model used in the mechanical simulation of the founding rope (adapted after Martínez \& Carroza, 1992). $W_{A H}$ : experimental tension in point $A\left(T_{1}\right), W_{f}$ : tension from the first float, $L_{C . F}$ : length of the mooring rope, $H$ : total depth, $h$ : depth of the main line with respect to sea bottom, $W_{\text {B.H. }}$ : resulting tension in point $B\left(T_{2}\right), X_{C . F}$ : excursion of the mooring $(I=1-6), \alpha_{1}, \alpha_{2}$ : theoretical attack angles of main line and mooring, $\mathrm{k}_{1}, \mathrm{k}_{2}, \mathrm{k}_{3}$ : constant distances. 


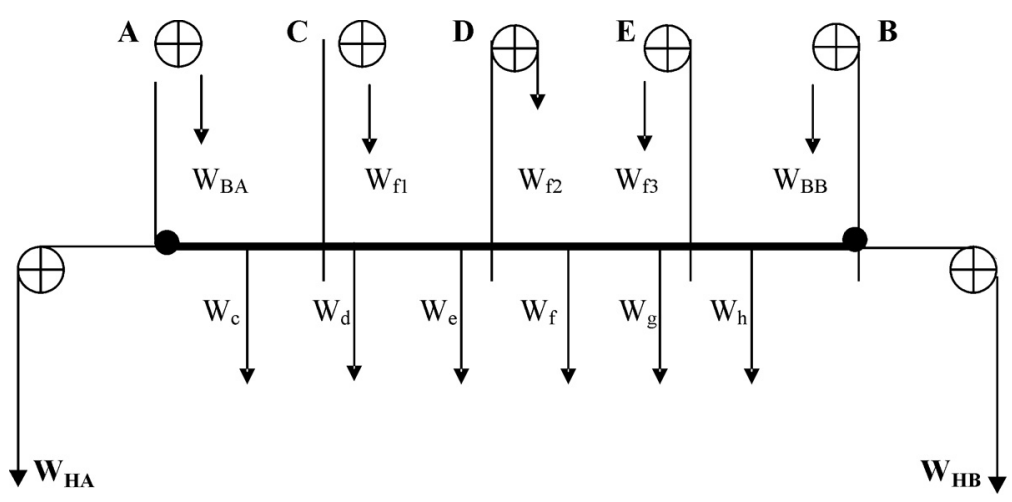

Figura 2. Esquema del modelo utilizado en la simulación mecánica de una sección de línea madre (adaptado de Trujillo \& Martínez, 2003). $\mathrm{W}_{\mathrm{BA}}$ : tensión de baliza $\mathrm{A}, \mathrm{W}_{\mathrm{BB}}$ : tensión de baliza $\mathrm{B}, \mathrm{W}_{\mathrm{HA}}$ : tensión horizontal de la línea madre. Terminal $A, W_{H B}$ : tensión horizontal de la línea madre. Terminal $B, W_{c}, W_{d}, W_{e}, W_{f}, W_{g}, W_{h}:$ pesos de los organismos en cultivo, $W_{\mathrm{f} 1}, W_{\mathrm{f} 2}, W_{\mathrm{f} 3}$ : fuerzas compensatorias del peso de los organismos en cultivo.

Figure 2. Diagram of the mechanical simulation model of a main line section (adapted from Trujillo \& Martínez, 2003). $W_{B A}$ : tension in buoy $A, W_{B B}$ : tension in bouy $B, W_{H A}$ : horizontal tension in the main line. End $A, W_{H B}$ : horizontal tension in the main line. End $B, W_{c}, W_{d}, W_{e}, W_{f}, W_{g}, W_{h}$ : live weight of cultured organisms, $W_{f 1}, W_{f 2}$, $W_{f 3}$ : compensatory forces of cultured organisms live weight.

que permita obtener una reducción significativa de la tensión resultante sobre el sistema de anclaje $\left(\mathrm{T}_{2}\right) \mathrm{y}$, por otra parte, configurar la línea madre con unidades de cultivo, determinando la forma y tensión de ella, producto de las fuerzas gravitacionales.

Para realizar las experiencias de simulación mecánica, se determinaron las siguientes variables:

Tensión en la línea madre $\left(\mathrm{T}_{1}\right)$ que se transmite hacia el sistema de fondeo, a partir de la tensión teórica calculada. De acuerdo a esto, para el estudio del comportamiento de la tensión resultante sobre el anclaje $\left(\mathrm{T}_{2}\right)$, se consideraron las distintas relaciones de aspecto del cabo de fondeo.

Relación de aspecto $\left(\mathrm{R}_{\mathrm{A}}\right)$, cociente entre la profundidad de la línea madre, respecto al fondo, y la longitud del cabo de fondeo. Las $\mathrm{R}_{\mathrm{A}}$ consideradas fueron: $1 / 2,5 ; 1 / 3,0 ; 1 / 3,5 ; 1 / 4,0 ; 1 / 4,5 ; 1 / 5,0$. Estas determinaron los ángulos de ataque en el anclaje $\left(\phi_{2}\right)$, que se forman entre la normal al sistema de anclaje y la tangente del cabo de fondeo, los cuales tienen importancia por su efecto sobre el poder de agarre del sistema de anclaje.

Proporción de recorte o excursionamiento de la línea madre $\left(\mathrm{P}_{\mathrm{R}}\right)$, relación entre la longitud de la cuerda (longitud de la línea madre) y el arco formado (flecha) por la distribución de su propio peso, cuya profundidad está determinada por las fuerzas gravitacionales ejercidas por el peso de los componentes de la línea de cultivo. La $\mathrm{P}_{\mathrm{R}}$ determina el ángulo de ataque de la catenaria $(\alpha)$, el cual se forma entre la tangente de la línea madre y la normal.

\section{Materiales y equipos para la simulación}

Se construyó un panel de prueba vertical, de estructura de madera y superficie de material de cartón piedra, de 2,44 x 1,22 m (superficie útil igual a 2,977 $\mathrm{m}^{2}$ ). Asimismo, se incorporó un juego de once poleas de $50 \mathrm{~mm}$ de diámetro, para el deslizamiento de los pesos. Estas permitieron configurar la unión entre la línea madre, primer cabo de flotadores y cabo de fondeo, y facilitaron la interacción entre los componentes de fuerza que se originan al variar la tensión inicial que actúa sobre la línea madre.

Para la simulación de las cargas hidrodinámicas que afectan al sistema de la línea de cultivo prototipo, se utilizaron cargas gravitacionales, con ayuda de recipientes de plástico liviano rellenos con arena de duna, bajo distintos excursionamientos. Para el primer modelo, relación de aspecto $\left(\mathrm{R}_{\mathrm{A}}\right)$; para el segundo modelo, proporción de recorte $\left(\mathrm{P}_{\mathrm{R}}\right)$ de la línea madre.

Las resultantes de las cargas gravitacionales sobre ambos modelos fueron medidas con ayuda de una balanza analítica de $1.200 \mathrm{~g}$ y precisión de $0,1 \mathrm{~g}$. Además, para cada uno de los juegos de mediciones de tensión-excursionamiento considerados, se evaluó 
el ángulo de ataque del cabo de fondeo respecto a la normal, con ayuda de un transportador circular en el punto $\mathrm{N}^{\circ} 1$ y uno semicircular, del tipo Tecnígrafo Giratorio, en el punto $\mathrm{N}^{\circ} 2$. Para el caso de la sección de la línea madre, se evaluó el ángulo de ataque formado entre la tangente de la línea madre y la normal, con ayuda de un transportador semicircular.

Conforme al tipo de material de fibra de polietileno a utilizar en la construcción de la línea de cultivo prototipo, sus propiedades físicas establecieron un patrón de hundimiento para toda la estructura, para relaciones de aspecto entre 2,5 y 5 veces, con una profundidad de línea madre de $8,5 \mathrm{~m}$. Asimismo, se estableció el patrón de hundimiento de una sección de línea madre, equivalente a la distancia entre dos balizas igual a $10 \mathrm{~m}$ de longitud, considerando el peso de seis unidades de cultivo, de acuerdo a los cálculos realizados para la etapa de desarrollo adulto de los organismos en cultivo.

\section{RESULTADOS}

Conforme a la metodología planteada, se determinó el peso total del sistema como la sumatoria de los pesos de los elementos constructivos que lo conforman, incluyendo organismos en cultivo. En tal sentido, se obtuvo el peso sumergido total de la línea de cultivo de $100 \mathrm{~m}$ igual a $579,77 \mathrm{~kg}$.

Asumiendo que la configuración adoptada por la línea madre entre dos flotadores se asemeja a una catenaria, se determinó la tensión mínima sobre la línea $\left(\mathrm{T}_{\mathrm{o}}\right)$ es igual a 47,13 kgf. La tensión que se produjo sobre el cabo de los flotadores, tensión en el extremo de la catenaria $(\mathrm{T})$, fue de 55,337 kgf, con una componente vertical de tensión $\left(\mathrm{T}_{\mathrm{y}}\right)$ igual a $27,66 \mathrm{kgf}$.
Sobre la base del valor de la tensión de $\mathrm{T}_{\mathrm{y}}$ se calculó la tensión máxima teórica $\left(\mathrm{T}_{\mathrm{y}}^{\prime}\right)$ igual a 41,49 kgf. Asimismo, se determinó la resistencia a la ruptura de los cabos, de acuerdo a Berteaux (1976), la cual fue igual a $249,65 \mathrm{kgf}$ y resultando para este propósito un diámetro de $3 / 8$ pulgadas.

Los flotadores de media agua a utilizar en la línea madre son de PVC expandido y denso, reutilizados y cuya flotabilidad se calculó teóricamente tomando en cuenta su volumen, densidad y flotabilidad. Se realizó una corrección de su flotabilidad teórica utilizando un factor de pérdida entre un 25 y $30 \%$. Por lo tanto, la flotabilidad del flotador requerida $\left(\mathrm{Q}_{\mathrm{fr}}\right)$ fue igual a $2 \mathrm{kgf}$.

\section{Resistencia hidrodinámica del sistema}

Se desarrolló la primera parte de la metodología que consistió en determinar la tensión, en forma teórica, ejercida por las cargas hidrodinámicas sobre la línea madre. Para ello se utilizó la ecuación (1) resultando una resistencia teórica total del sistema igual a 777,15 kgf. La resistencia de cada uno de los componentes de la línea de cultivo se observa en la Tabla 1.

\section{Dimensionamiento del sistema de anclaje}

Utilizando la resistencia teórica total de sistema, se determinó la influencia de la tensión en la línea madre ejercida sobre el cabo de fondeo, mediante el cálculo de la resistencia del cabo de fondeo al flujo $\left(\mathrm{R}_{\mathrm{cf} .}=0,536 \mathrm{kgf} \cdot \mathrm{m}^{-1}\right)$, el peso del cabo en el agua $\left(\mathrm{P}_{\mathrm{ch}}=0,0447 \mathrm{~kg} \cdot \mathrm{m}^{-1}\right)$ y el ángulo de ataque crítico del cabo $\left(\Phi_{\mathrm{c}}=73,51^{\circ}\right)$.

Conociendo los valores de flotabilidad del primer flotador-baliza $\left(\mathrm{Q}_{\mathrm{fl}}=128 \mathrm{kgf}\right)$, peso de la primera linterna $\left(\mathrm{P}_{\mathrm{cl}}=8,78 \mathrm{~kg}\right)$ y tensión en línea madre en

Tabla 1. Cálculos teóricos de resistencia de los componentes de la línea de cultivo de ostra perla. Re: número de Reynolds, $C_{R}$ : coeficiente resistivo, $F$ : superficie expuesta al flujo, $R_{I}$ : resistencia hidrodinámica teórica.

Table 1. Theoretical computation of longline resistance components for pearl oyster culture. Re: Reynolds number, $C_{R}$ : resistance coefficient, $F$ : area subject to flow, $R_{I}$ : theoretical hydrodynamic resistance.

\begin{tabular}{lcccccccc}
\hline \multicolumn{1}{c}{ Sección } & $\mathbf{N}^{\circ}$ & $\begin{array}{c}\text { Longitud } \\
(\mathbf{m})\end{array}$ & $\begin{array}{c}\text { Diámetro } \\
\mathbf{( m )}\end{array}$ & $\begin{array}{c}\text { Velocidad } \\
\text { corriente } \\
(\mathbf{m} / \mathbf{s})\end{array}$ & $\mathbf{R e}$ & $\mathbf{C}_{\mathbf{R}}$ & $\begin{array}{c}\mathbf{F} \\
\left(\mathbf{m}^{2}\right)\end{array}$ & $\begin{array}{c}\mathbf{R}_{\mathbf{I}} \\
(\mathbf{k g f})\end{array}$ \\
\hline Línea madre & 1 & 100,0 & 0,019 & 0,425 & $8,3 \times 10^{3}$ & 1,41 & 1,900 & 25,23 \\
Cabo de fondeo & 2 & 30,0 & 0,019 & 0,425 & $8,3 \times 10^{3}$ & 1,41 & 0,570 & 15,14 \\
Cabo de flotadores & 10 & 1,5 & 0,001 & 0,425 & $3,5 \times 10^{3}$ & 1,40 & 0,015 & 1,58 \\
Linternas & 66 & 1,8 & 0,450 & 0,425 & $19,7 \times 10^{4}$ & 1,46 & 0,810 & 735,20 \\
\hline
\end{tabular}


el Punto $1\left(T_{1}=388,57 \mathrm{kgf}\right)$, se determinó la tensión transmitida hacia el cabo de fondeo. La resultante de tensión en el Punto 1, del vector $\mathrm{t}_{1}$, fue de 786,24 kgf y el ángulo de ataque del vector $\mathrm{t}_{1}\left(\Phi_{1}\right)$ fue igual a $89,55^{\circ}$. Posteriormente, se determinó la tensión que se transmite hacia el sistema de anclaje en el punto $2\left(t_{2}\right)$, la cual fue igual a 740,44 kgf.

\section{Elemento de anclaje propuesto}

El elemento de fondeo seleccionado para la línea de cultivo fue el ancla "tipo rezón" de 5 uñas. Este cumple con las características dadas por Wiegel (1964), para su uso en diversos tipos de fondo, que son: adecuada velocidad de entierro para desarrollar una buena resistencia, profundidad de entierro de sus uñas para evitar que el ancla se desplace, capacidad de penetrar fondos duros, estabilidad o libertad de balanceo, bastante peso muerto en el caso de sustratos de bajo agarre y fácil de fabricar. Para el cálculo del poder de agarre $\left(\mathrm{P}_{\mathrm{a}}\right)$ se utilizó la ecuación (11), resultando un peso de anclaje de $898,8 \mathrm{~kg}$, para un peso seco igual a $60 \mathrm{~kg}$.

\section{Accesorios del sistema}

La determinación se realizó sobre la base de dos parámetros, de acuerdo a las recomendaciones de Martínez et al. (1990):

Carga ambiental: Para una carga máxima de trabajo en un extremo de la línea madre igual a $3.885,75 \mathrm{kgf}$ se aplicó un factor de seguridad igual 5 , se seleccionaron grilletes galvanizados tipo corazón de $3 / 4$ y $5 / 16$ pulgadas de diámetro.

Carga por manejo: Las mayores tensiones que surgen en el cabo orinque de fondeo se producen cuando se hace el garreo del anclaje junto con la línea madre y las unidades de cultivo. Por cuanto estas tensiones aún no han sido medidas in situ, se procedió a hacer un cálculo aproximado considerando que la embarcación a utilizar es del tipo "peñero", con dos motores fuera de borda, con una potencia de $80 \mathrm{HP}$ en total. A tal efecto, se calculó la tracción de acuerdo a Hamuro (1964 en Okonski \& Martini, 1987), quien indica que la potencia empleada en las maniobras puede ser transformada en fuerza ( $\mathrm{kgf} ; \mathrm{kNw}$ ), considerando que estas embarcaciones desarrollan entre 14 y 18 libras por BHP. Por lo tanto, la fuerza de tracción calculada, en este caso, fue de $6.000 \mathrm{~kg}$.

Para la unión entre el cabo de fondeo, línea madre y primer cabo de flotador, se utilizó una argolla de acero galvanizada de 3/4" de diámetro, donde se conectaron los grilletes de los cabos anteriormente citados. Esta argolla facilitó la desconexión de alguno de los cabos de la unión, para efectos de reparación, reorientación de líneas, puntos de amarre de sensores para el estudio de tensión, etc.

\section{Configuración del cabo de fondeo y sección de línea madre}

Para realizar la experiencia sobre el panel de prueba se realizó el siguiente procedimiento:

Cabo de fondeo: Considerando las recomendaciones de Baranov (1969) y Fridman (1981), se utilizó una cadena metálica para simular el cabo de fondeo. Sus terminales se fijaron en los apoyos A y B en forma asimétrica (Fig. 1). Al extremo del apoyo A se unieron dos hilos flexibles ligero, uno dirigido hacia una polea, en cuyo extremo se unió un recipiente para determinar la resistencia del sistema (componente horizontal) y el otro hilo, pasando a través de dos poleas, con la finalidad de simular la componente vertical, representó una fuerza equivalente a la fuerza de empuje de la baliza prototipo, que varió de acuerdo a los excursionamientos. El terminal B de la cadena, unido a un hilo flexible de PA, se dirigió hacia una cuarta polea para determinar la componente horizontal, generada por el anclaje.

La tensión teórica calculada $\left(T_{1}=777,15 \mathrm{kgf}\right)$ en el sistema de línea de cultivo fue tomada como referencia para la determinar la configuración del cabo de fondeo bajo diferentes $\mathrm{R}_{\mathrm{A}}$, mediante la simulación de la fuerza que se transmite hacia el sistema de fondeo. Por lo tanto, se utilizó:

Factor de escala lineal: considerando como unidad prototipo la longitud del cabo de fondeo $\left(\mathrm{L}_{\mathrm{n}}\right)$, bajo distintas $\mathrm{R}_{\mathrm{A}}(1 / 2,5 ; 1 / 3,0 ; 1 / 3,5 ; 1 / 4,0 ; 1 / 4,5 ; 1 / 5,0)$, correspondientes a 53,125; 63,7; 74,37; 85; 95,625 y $106,25 \mathrm{~cm}$, el factor de escala lineal $\left(\mathrm{C}_{\mathrm{L}}\right)(12)$ fue igual a 40.

Factor de escala de peso: considerando el peso de la longitud del cabo de fondeo como unidad prototipo $\left(F_{n}\right)$, bajo distintas $R_{A}(1 / 2,5 ; 1 / 3 ; 1 / 3,5 ; 1 / 4 ; 1 / 4,5$; $1 / 5)$ y un peso 13,$08 ; 16,073 ; 18,384 ; 21,587 ; 23,51$ $\mathrm{y}$ de $25,00 \mathrm{~g}$, el factor de escala de fuerza $\left(\mathrm{C}_{\mathrm{R}}\right)(13)$ fue de $9.776,3$.

Sección de línea madre: Los terminales de la cadena se fijaron en los apoyos A y B en forma simétrica (Fig. 2). Al extremo A se unieron dos hilos flexibles ligeros de PA, uno dirigido hacia una polea, en cuyo 
extremo se unió un recipiente para determinar la componente horizontal (tensión de la línea madre) y el otro se dirigió hacia la otra polea, para simular el componente vertical, que representó una fuerza equivalente a la fuerza de empuje de la baliza superficial prototipo. El otro terminal B de la cadena, se consideró simétrico al punto A, de acuerdo a Trujillo \& Martínez (2003).

Entre A y B se fijaron tres puntos de amarre (C, D, E) con ayuda de hilos flexibles ligeros, los cuales, deslizándose a través de tres poleas, determinaron las componentes verticales, cuyas fuerzas son generadas por las boyas de media agua para compensar los pesos de los organismos en cultivo (c, d, e, f, g, h) que forman una catenaria (Fig. 2).

Factor de escala lineal: se utilizó como unidad prototipo la longitud de una sección de línea madre $\left(\mathrm{L}_{\mathrm{n}}\right)$, equivalente a $10 \mathrm{~m}$, correspondiente a $53 \mathrm{~cm}$ en el modelo, el factor de escala lineal $\left(\mathrm{C}_{\mathrm{L}}\right)$ fue de 18,86 .

Factor de escala de peso: se consideró como unidad prototipo el peso de la sección de línea madre $\left(\mathrm{F}_{\mathrm{n}}\right)$, bajo la acción de los pesos máximos igual a $47,016 \mathrm{~g}$, el factor de escala de fuerza $\left(\mathrm{C}_{\mathrm{R}}\right)$ fue de $1.233,13$.

El elemento de carga, arena de duna, fue vertido en cada uno de los recipientes, lo cual permitió desplazar los puntos de amarre $(\mathrm{C}, \mathrm{D}, \mathrm{E})$ de la cadena en sentido vertical hasta coincidir con la cuerda (L) de la catenaria; y los terminales de la cadena, en sentido horizontal, hasta coincidir con el centro de la coordenada en los puntos de apoyo (A) y (B) (Fig. 2). De esta forma, las fuerzas aplicadas en los extremos y centro de la cadena fueron las propias reacciones de las componentes horizontales y verticales, respectivamente.

Basados en los resultados obtenidos en las pruebas de simulación, se configuró la catenaria para seis linternas, en función del peso de ellas con organismos en fase de adulto (Fig. 3).

De acuerdo a las medidas estándares de la línea de cultivo "Margarita", utilizado como prototipo, se determinó que las linternas $\mathrm{N}^{\circ} 3$ y No 4 , sin la compensación vertical de la flotación $(\mathrm{C}, \mathrm{D}, \mathrm{E})$, alcanzaron una profundidad de operación de 7,37 m (Fig. 3), correspondiente a una proporción de recorte promedio del $93,6 \%$ y un ángulo de ataque de $55,86^{\circ}$. En tal sentido, para una correcta instalación se requiere una profundidad de operación límite de 4,35 $\mathrm{m}$ y un ángulo de ataque de $90^{\circ}$. Estas últimas condiciones permitirían a las unidades de cultivo trabajar a la profundidad antes mencionada y conservar la distancia pre-establecida entre ellas, para evitar interferirse. Por lo tanto, la profundidad máxima será igual a:

$$
\mathrm{H}_{\text {Max.Cultivo }}=\mathrm{h}_{\mathrm{b}}+\mathrm{h}_{\text {chicote }}+\mathrm{h}_{\text {bridas }}+\mathrm{h}_{\text {Linterna }}
$$

donde:

$\mathrm{h}_{\mathrm{b}} \quad$ : longitud bajante de baliza $=1,5 \mathrm{~m}$

$\mathrm{h}_{\text {chicote }}:$ longitud cabo de linterna $=0,5 \mathrm{~m}$

$\mathrm{h}_{\text {bridas }} \quad$ : longitud bridas linterna $=0,556 \mathrm{~m}$

$\mathrm{h}_{\text {Linterna }}:$ lltura de la linterna $=1,8 \mathrm{~m}$

Con el peso total de una sección de $10 \mathrm{~m}$ de línea madre prototipo igual a $57,977 \mathrm{~kg}$ y una tensión horizontal en el terminal de la catenaria igual

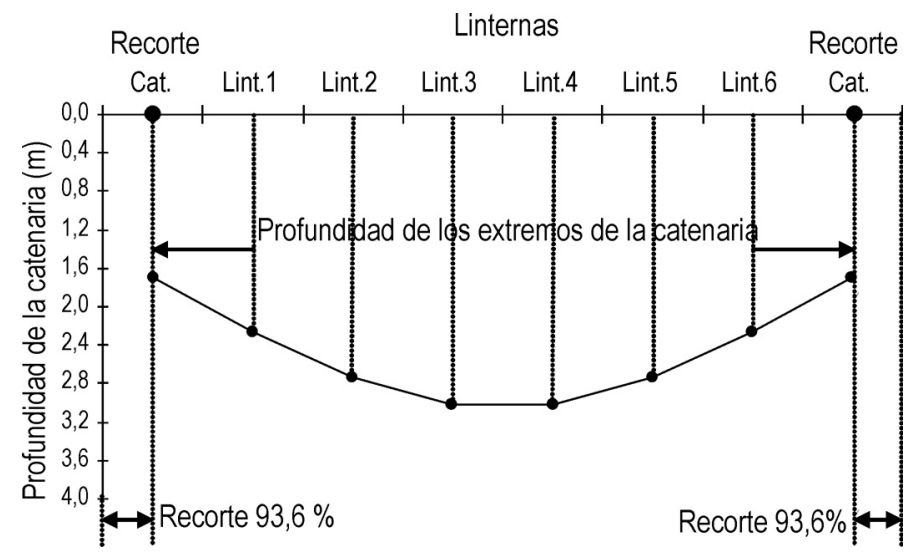

Figura 3. Configuración experimental de una sección de línea madre. Ángulo de ataque 55,86

Figure 3. Experimental configuration of a main line section. Attack angle $55.86^{\circ}$. 
a 55,337 kgf, se realizaron diez mediciones en el panel de simulación a fin de determinar los valores promedio de tensión en la línea madre del prototipo en el apoyo A (baliza 1) y apoyo B (baliza 2) (Tabla 2). El comportamiento de la línea madre se indica en la Figura 4.

Por otra parte, habiendo determinado el peso de la línea de fondeo bajo diferentes valores de excursionamiento, en base a diez mediciones en el panel de simulación, se determinaron los valores promedio de tensión en el terminal de la línea de fondeo del prototipo, en el punto de apoyo B (Tabla $3)$. Estos valores muestran una relación inversa con los diferentes valores de excursionamiento (tasa de recorte) (Fig. 5), para una tensión de prueba constante de $388,57 \mathrm{kgf}$.

En la Figura 5 se observa que la diferencia de tensión que se produce entre ambos puntos conside- rados en la línea de cultivo, es decir, entre la unión de la línea madre y cabo de fondeo $\left(\mathrm{T}_{1}\right)$ y la unión del cabo de fondeo con el anclaje $\left(T_{2}\right)$, permite establecer que si bien esta ocurre en todas las relaciones de aspecto evaluadas, se observa una disminución de tensión desde la línea madre hacia el sistema de anclaje, lográndose mínimas tensiones resultantes en este último cuando se emplea $\mathrm{R}_{\mathrm{A}}$ de $1 / 4$ y $1 / 5$. No obstante, se apreció un incremento en el valor de $T_{2}$ respecto a $T_{1}$ en la relación $R_{A} 1 / 4,5$. Por lo tanto, al considerar esta disminución de la tensión hacia el cabo de fondeo, su longitud debería ser cuatro o cinco veces la profundidad de la línea madre respecto al fondo, cuyos valores serían menor que la tensión en la línea madre de prueba. La tensión de la flotabilidad del primer flotador, disminuye conforme aumenta la relación de aspecto, registrándose una tensión mínima cuando $R_{A}$ es igual a 1/5 (Tabla 3 ).

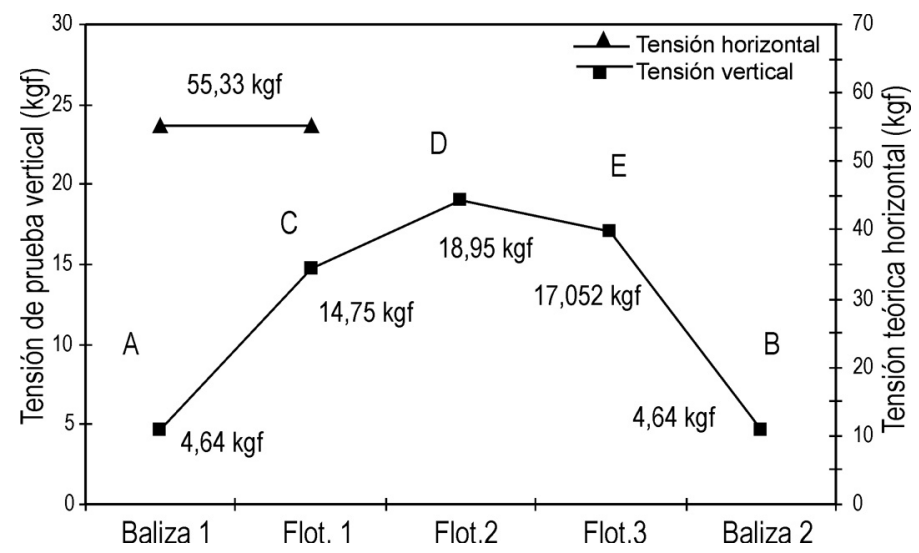

Figura 4. Tensión experimental sobre la catenaria. Ángulo de ataque $55,86^{\circ}$.

Figure 4. Experimental tension upon the catenary. Attack angle $55.86^{\circ}$.

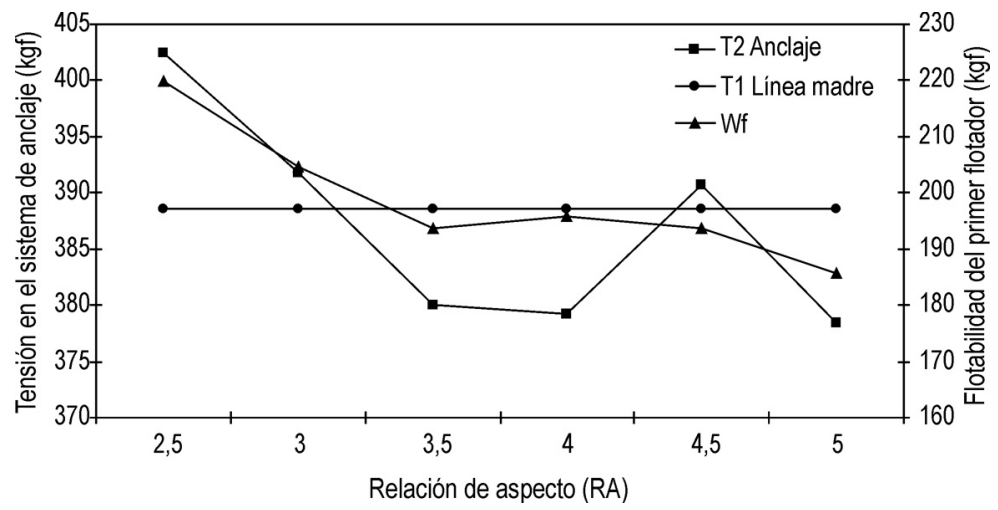

Figura 5. Tensión en sistema de fondeo. Tensión de prueba $388,57 \mathrm{kgf}$.

Figure 5. Tension of mooring system. Test tension $388.57 \mathrm{kgf}$. 
Por otra parte, al evaluar los ángulos de ataque bajo distintas $\mathrm{R}_{\mathrm{A}}$ (Fig. 6), ante un valor de tensión de prueba en la línea madre igual a $388,57 \mathrm{kgf}$, indican una relación directa con los valores de ángulos de ataque de fondeo teórico $\left(\alpha_{2}\right)$ y de línea madre $\left(\alpha_{1}\right)$, los cuales tienden a aumentar a medida que se incrementa dicha relación (Tabla 4).

\section{DISCUSIÓN}

El análisis teórico de la resistencia hidrodinámica del sistema se realizó suponiendo un ángulo de ataque de $90^{\circ}$ de la estructura respecto al flujo de agua, a fin de facilitar el cálculo preliminar. Este se realizó en función de las cargas cuasi-estáticas en virtud a

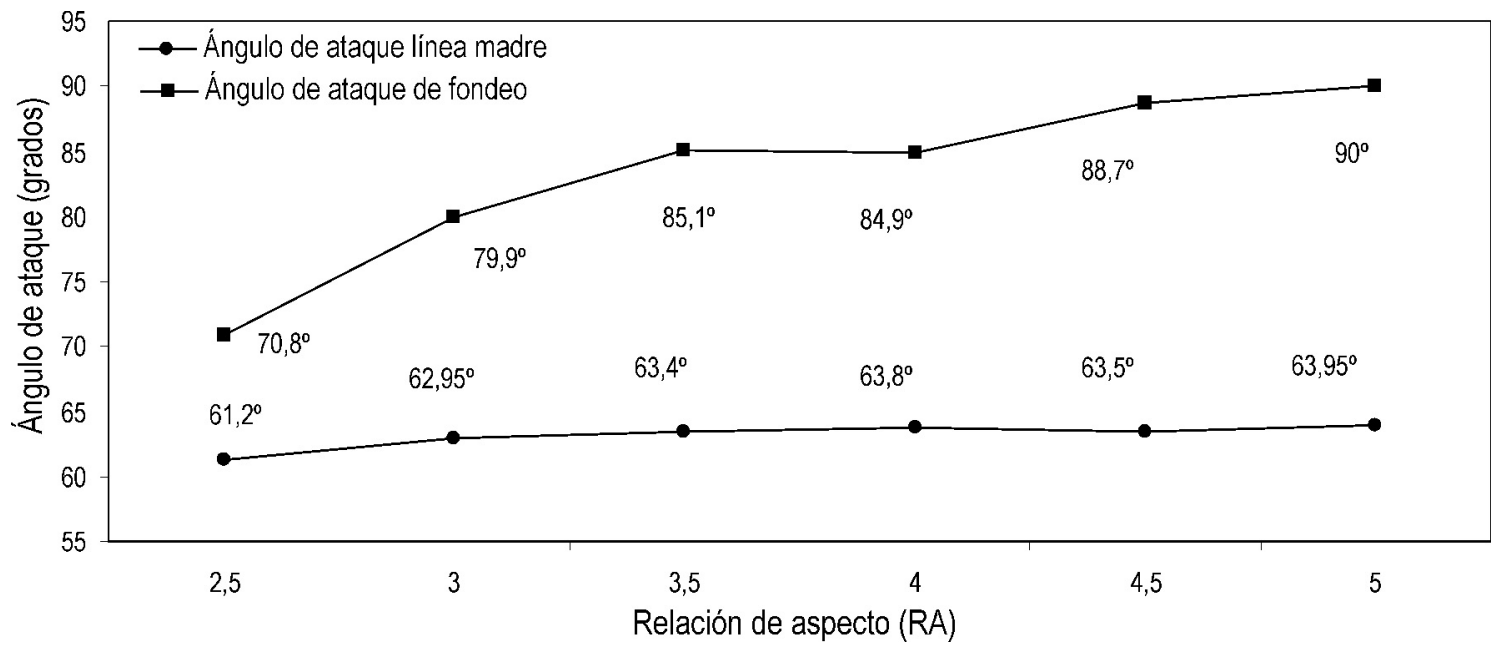

Figura 6. Ángulo de ataque de fondeo teórico. Tensión $388,57 \mathrm{kgf}$.

Figure 6. Theoretical attack angle of mooring. Tension $388.57 \mathrm{kgf}$.

Tabla 2. Tensión experimental en la catenaria de una sección de la línea madre. DE: desviación estándar.

Table 2. Experimental tension upon the catenary of a main line section. DE: standard deviation.

\begin{tabular}{cccccccccc}
\hline \multicolumn{2}{c}{ Baliza A } & \multicolumn{2}{c}{ Flotación 1 } & \multicolumn{2}{c}{ Flotación 2 } & \multicolumn{2}{c}{ Flotación 3 } & \multicolumn{2}{c}{ Baliza B } \\
\hline $\begin{array}{c}\text { Tensión } \\
\text { kgf }\end{array}$ & $\begin{array}{c}\mathrm{DE} \\
\pm\end{array}$ & $\begin{array}{c}\text { Tensión } \\
\mathrm{kgf}\end{array}$ & $\begin{array}{c}\mathrm{DE} \\
\pm\end{array}$ & $\begin{array}{c}\text { Tensión } \\
\mathrm{kgf}\end{array}$ & $\begin{array}{c}\mathrm{DE} \\
\pm\end{array}$ & $\begin{array}{c}\text { Tensión } \\
\mathrm{kgf}\end{array}$ & $\begin{array}{c}\mathrm{DE} \\
\pm\end{array}$ & $\begin{array}{c}\text { Tensión } \\
\mathrm{kgf}\end{array}$ & $\begin{array}{c}\mathrm{DE} \\
\pm\end{array}$ \\
\hline 4,64 & 0,57 & 14,75 & 0,95 & 18,95 & 0,79 & 17,05 & 1,18 & 4,64 & 1,28 \\
\hline
\end{tabular}

Tabla 3. Tensión experimental en el cabo de fondeo entre el anclaje y primer flotador. $R_{A}:$ relación de aspecto, $L$ : longitud, DE: desviación estándar.

Table 3. Experimental tension upon the mooring rope between the mooring and the first float. $R_{\mathrm{A}}$ : aspect ratio, L: length, DE: standard deviation.

\begin{tabular}{cccccc}
\hline $\mathbf{R}_{\mathbf{A}}$ & $\mathbf{L}$ & $\begin{array}{c}\text { Tensión } \\
\text { terminal-anclaje }\end{array}$ & $\mathbf{D E}$ & $\begin{array}{c}\text { Tensión } \\
\text { terminal-primer flotador }\end{array}$ & DE \\
\hline $\mathrm{h} \cdot(\mathrm{n} \cdot \mathrm{h})$ & $(\mathrm{m})$ & $(\mathrm{Kgf})$ & \pm & $($ Kgf $)$ & \pm \\
$1 / 2,5$ & 21,25 & 402,86 & 0,667 & 219,89 & 0,732 \\
$1 / 3,0$ & 25,50 & 391,18 & 0,962 & 204,63 & 0,367 \\
$1 / 3,5$ & 29,75 & 380,05 & 0,464 & 193,65 & 0,601 \\
$1 / 4,0$ & 34,00 & 379,27 & 0,306 & 195,81 & 0,451 \\
$1 / 4,5$ & 38,25 & 390,64 & 0,705 & 193,59 & 0,547 \\
$1 / 5,0$ & 42,50 & 378,40 & 0,576 & 185,59 & 0,500 \\
\hline
\end{tabular}


Tabla 4. Ángulos de ataque experimentales en la línea de fondeo. $\mathrm{R}_{\mathrm{A}}$ : relación de aspecto, $\mathrm{L}$ : longitud, DE: desviación estándar.

Table 4. Experimental attack angles on the mooring line. $R_{A}$ : aspect ratio, $L$ : length, $D E$ : standard deviation.

\begin{tabular}{cccccc}
\hline $\mathbf{R}_{\mathbf{A}}$ & $\mathbf{L}$ & $\begin{array}{c}\text { Ángulo de ataque } \\
\text { terminal- anclaje } \\
(\alpha)\end{array}$ & $\mathbf{D E}$ & $\begin{array}{c}\text { Ángulo de ataque } \\
\text { Terminal-línea madre } \\
(\boldsymbol{\alpha})\end{array}$ & $\mathbf{D E}$ \\
\hline $\mathrm{h} \cdot(\mathrm{n} \cdot \mathrm{h})$ & $(\mathrm{m})$ & $($ grados $)$ & \pm & $($ grados $)$ & \pm \\
$1 / 2,5$ & 21,25 & 61,20 & 0,580 & 70,8 & 0,918 \\
$1 / 3,0$ & 25,50 & 62,95 & 0,158 & 79,9 & 0,316 \\
$1 / 3,5$ & 29,75 & 63,45 & 0,685 & 85,1 & 0,737 \\
$1 / 4,0$ & 34,00 & 63,80 & 0,349 & 84,9 & 0,994 \\
$1 / 4,5$ & 38,25 & 63,50 & 0,520 & 88,7 & 0,483 \\
$1 / 5,0$ & 42,50 & 63,95 & 0,368 & 90,0 & - \\
\hline
\end{tabular}

las características de la estructura y del medio. Para ello se utilizó el concepto de resistencia total del sistema sobre la base de la ecuación de resistencia de Fridman (1981).

Para el dimensionamiento del sistema y su comportamiento hidrodinámico, se tomaron en cuenta las variables de fuerzas generadas por la corriente y marea predominantes en la zona. Estas fueron obtenidas in situ a través de un seguimiento anual de la velocidad y dirección, incorporando el vector resultante en el cálculo. La velocidad, así como los otros parámetros jugaron un rol importante en la ecuación, por cuanto sus aproximaciones permitieron determinar el coeficiente de resistencia $\left(\mathrm{C}_{\mathrm{R}}\right)$ para cada uno de los elementos constructivos del sistema y para el sistema en general. En esta ecuación no se consideraron las cargas por efecto de las olas debido a la baja ocurrencia en bahía de Charagato.

Por otra parte, al trabajar con modelos de sistemas flexibles, se dificulta la reproducción en laboratorio de las condiciones de trabajo del prototipo con sus respectivas variables. No obstante, la facilidad con que se pone en práctica el método de simulación mecánica, además del ahorro de tiempo y energía, y la dificultad que representa la experimentación directa, constituye una herramienta útil y sencilla para comprender y visualizar la forma en que operan las estructuras flexibles inmersas en el mar (Martínez \& Carroza, 1992). Por ello, este procedimiento, a pesar de tener estas limitantes, permitió a través de varios excursionamientos definir la forma y tensión del cabo de fondeo para mejorar el rendimiento del sistema de anclaje. Del mismo modo, se pudo definir la forma y tensión en los extremos de la línea madre, a través de diferentes proporciones de recorte, con la finalidad de lograr la sustentación óptima de la línea madre sujeta a cargas gravitatorias producidas por el peso del sistema. De acuerdo a Carroza (1990), estas cargas pueden tener menor efecto sobre la resultante que se transmite hacia el fondeo, si el sistema de flotación de media agua es dimensionado correctamente, para compensar el peso del sistema.

Con relación al análisis de la configuración del sistema de fondeo y de una sección de la línea madre, al utilizar los procedimientos aplicados por Martínez \& Carroza (1992) y Trujillo \& Martínez (2003), se determinó que los resultados obtenidos en el presente estudio se asemejan con los obtenidos por estos autores, en cuanto a la tendencia a la disminución de los valores de tensión en el cabo de fondeo en función del incremento de la RA, aun cuando habían diferencias en cuanto a condiciones del medio y componentes del diseño. En este sentido, al observar las tensiones en el sistema de anclaje $\mathrm{T}_{2}$, estas fueron mayores que la tensión de prueba $T_{1}(388,57 \mathrm{kgf})$ a partir de $\mathrm{R}_{\mathrm{A}}$ $1 / 2,5$ a $1 / 3$, y disminuyeron cuando se emplearon $R_{A}$ de $1 / 3,5 ; 1 / 4$ y $1 / 5$.

Martínez \& Carroza (1992) también encontraron una relación semejante entre estas dos variables, aun cuando el valor de la tensión en el sistema de anclaje $\left(\mathrm{T}_{2}\right)$ fue siempre menor que las tensiones de prueba $\left(\mathrm{T}_{1}\right)$ seleccionadas y aplicadas en la línea madre en todas las relaciones de aspecto. La mínima tensión resultante encontrada sobre el sistema de anclaje se logró al emplear $\mathrm{R}_{\mathrm{A}}$ de 1/3,5 y 1/5,5.

De acuerdo a Trujillo \& Martínez (2003) la tensión en una sección de línea madre, cuando se modela, muestra una tendencia a aumentar a medida que aumenta la proporción de recorte, y en la misma forma, los ángulos de ataque. En tanto que la forma 
de la línea madre en el presente trabajo, utilizando tensiones constantes en los extremos de la sección analizada, producto de las fuerzas de tensión de la línea madre, se comportó como una catenaria al no existir una compensación de los pesos ejercidos por las cargas gravitatorias, producto del peso por unidad de longitud.

Considerando que la finalidad de esta investigación era compensar las fuerzas gravitatorias, transformando la línea catenaria en una línea recta con ayuda de fuerzas de flotación intermedia, adicionales a los puntos de sujeción en los extremos de la sección, que representaban la balizas superficiales, se pudo determinar que las tensiones verticales en dichos extremos tienden a disminuir sustancialmente, en tanto que las componentes horizontales permanecen constantes. Asimismo, la compensación total vertical en la sección de línea madre analizada, producto de la fuerza de flotación de los flotadores intermedios, fue igual a las fuerzas de inmersión de la misma sección, lo cual concuerda con lo expresado por Carroza (1990) en cuanto a las ventajas del buen diseño del sistema de flotación de media agua, para disminuir el efecto sobre la resultante que se transmite hacia el fondeo.

Cabe señalar, que Raman-Nair \& Colbourne (2003) aplicaron la técnica de modelamiento numérico para establecer un método de análisis de la dinámica de la línea principal de un sistema de cultivo de mejillones, basados en el formulación de Kane y presentar resultados de simulaciones de predicción, tanto de las fuerzas como de la forma que adoptan las líneas ante varios escenarios de cargas hidrodinámicas. Ellos asumieron que éstas son causadas por efecto de los pesos adheridos a la línea y por la resistencia viscosa. En este sentido, tomaron como carga directa una velocidad de fluido arbitrario y un campo de aceleración, el cual se asume que no es alterado por el sistema. Esto les permitió incluir los efectos de las olas y las corrientes.

Asimismo, estos autores para modelar las cargas en la línea madre, causadas por las trenzas de mejillones, utilizaron supuestos simples sobre la distribución de la masa de estos organismos y sus coeficientes de resistencia asociados. El modelo fue adaptado de una formulación más general propuesta por Rama-Nair \& Baddour (2002) para el modelamiento de sistemas de anclaje alejados de la costa. Este modelo lo consideraron útil para predecir las cargas que tienen importancia práctica para las instalaciones de cultivos de moluscos a pequeña escala.

\section{AGRADECIMIENTOS}

El presente trabajo fue realizado gracias al financiamiento del FONACIT en el marco del proyecto $\mathrm{N}^{\circ}$ 2000001360 "Dimensionamiento y construcción de un sistema de una línea de cultivo para moluscos bivalvos Ostra perla (Pinctada imbricata) y Mejillón (Perna perna) en la bahía de Charagato, isla de Margarita, Venezuela". Asimismo, un agradecimiento a José Alió por la lectura crítica del manuscrito y la traducción al inglés del resumen del artículo.

\section{REFERENCIAS}

Andreyev, N.N. 1962. Manual de artes y aparejos de pesca de material de redes. Pishepromuizdat, Moscú, 504 pp. (en ruso).

Baranov, F.I. 1939. Aplicación del método de simulación mecánica en el diseño de artes de pesca. Trudy Mosrybtuza, Vyp., 2: 103-130 (en ruso).

Baranov, F.I. 1966. Trabajos escogidos. Tomo I. Técnica de la pesca industrial. Industria Alimenticia, Moscú, 720 pp. (en ruso).

Baranov, F.I. 1969. Trabajos escogidos. Tomo I. Técnicas de la pesca industrial. Industria Alimenticia, Moscú, 719 pp. (en ruso).

Berteaux, H. 1976. Buoy engineering. John Wiley \& Sons, New York, 305 pp.

Carroza, L. 1990. Determinación de una metodología para la selección y dimensionamiento de un sistema para un longline de cultivo. Tesis de Pesquerías. Escuela de Ciencias del Mar, UCV, 194 pp.

Díaz, O., L. Troccoli \& J. Millán. 1998. Ensayos de cultivo de la ostra de mangle Crassostrea rhizophorae en la isla de Cubagua, Venezuela. Caribbean J. Sci., 34(3-4): 243-249.

Fridman A.L. 1981. Teoría y diseño de sistemas de pesca industriales. Industria Liviana y Alimenticia, Moscú, 327 pp. (en ruso).

Fridman, A.L. 1986. Calculations for fishing gears designs. FAO Manuals by Fishing News Books. Farnham Surrey, England, pp. 70-87.

Kondratyev, V.N. 1973. Modelación de artes de pesca industrial por el método de analogía mecánica. Industria Alimenticia, Moscú, 152 pp. (en ruso).

León, J., L. León \& J. Millán. 1998. Cultivo suspendido del mejillón Perna perna L.1758 (Mollusca: Bivalvia) en la isla de Cubagua, Venezuela. Caribb. Mar., 6: 12-18.

León L. 1997. Estudio de la comunidad de moluscos asociadas a los bancos de la ostra perlífera Pinctada 
imbricata de la isla de Cubagua, Venezuela. Memorias del II CLAMA, pp. 72-74.

León, L. \& J. Millán. 1996. Variación del índice de condición y de la composición bromatológica de la ostra perlífera Pinctada imbricata Roding, 1798 (Mollusca: Bivalvia) de las Cabeceras, isla de Cubagua, Venezuela. Mem. Soc. Ciens. Nat. La Salle, 56(146): 23-31.

León, L. \& E. Pereyra. 1994. Estudio sobre la biomasa específica del crecimiento, reproducción y otros comportamientos en la ostra perlífera Pinctada imbricata Roding, 1798 (Mollusca: Bivalvia) en el banco de las Cabeceras, isla de Cubagua, Venezuela. Memorias del III Congreso de Ciencias del Mar, 12 pp.

León, L., T. Cabrera \& L. Troccoli. 1987. Fijación e índice de engorde de la ostra perla Pinctada imbricata Roding, 1798 (Mollusca: Bivalvia) en tres bancos naturales del nororiente de Venezuela. Contrib. Cient. Ctro. Invest. Cient. UDO, 12: 42 pp.

Lodeiros, C. 1996. Influence des facteurs environnementaux sur la croissance du pétoncle tropical Euvola (Pecten) ziczac (L. 1758) cultivé en suspension au golfo de Cariaco. Venezuela. Tesis Ph.D., Universidad Laval, Canada, 112 pp.

Lodeiros, C. \& J.H. Himmelman. 1994. Relation among environmental conditions and growth and growth in the scallop Euvola (Pecten) ziczac (L.) in suspended culture. Aquaculture, 119:345-358.

Lodeiros, C. 1999. Factibilidad biológica del cultivo de especies de bivalvos marinos en el oriente de Venezuela. Memorias del Taller Venezolano sobre Aprovechamiento y Comercialización de Moluscos Bivalvos, pp. 12-17.

Lodeiros, D. P., A. Prieto, N. Narváez \& A. Guerra. 2002. Growth and survival of the pearl oyster Pinctada imbricata (Röding 1758) in supended and bottom culture in the Golfo de Cariaco, Venezuela. Aquac. Int., 10: 327-338.

Lodeiros, C. \& N. García. 2004. The use of sea urchins to control fouling during suspended culture of bivalves. Aquacult. Int., 203: 293-298

Martínez, G., T. Melo \& L. Carroza. 1990. Dimensionamiento y selección de componentes para un longline de cultivo de ostión del norte en la zona de Mejillones: I Parte. Dimensionamiento teórico. Estud. Doc., Universidad Católica Valparaíso, 4/90: $114 \mathrm{pp}$.

Martínez, G. \& L. Carroza. 1992. Configuración del cabo de fondeo de un longline de cultivo a través de simulación análoga. Invest. Mar., Valparaíso, 20: 55-66.

Miyazaki, Y. 1970. The configuration and tension of a rope and plane net set in an uniform stream. J. Tokyo Univ. Fish., 56(1-2): 49-117.

Neshyba, S \& T. Fonseca. 1981. Corrientes costeras. Manual de mediciones y análisis. Invest. Mar. Supl., 7: 49-69.

Ogg, R. 1969. Anchors. In: J. Myers, C. Holm \& R. McAilster (eds.). Handbook of ocean and underwater engineering. Mc Graw-Hill, New York, Section, 4: 70-74.

Okonski, S.L. \& L.W. Martini. 1987. Artes y métodos de pesca. Materiales didácticos para la capacitación técnica. Hemisferio Sur, 339 pp.

Rama-Nair, W. \& R.E. Baddour. 2002. Three dimensional coupled dynamics of a buoy and multiple mooring lineas: formulation and algorithm. Quart. J. Mech. Appl. Math., 55(2): 179-207.

Raman-Nair, W. \& B. Colbourne. 2003. Dynamics of a mussel longline system. Aquacult. Eng., 27(3): 191-212.

Troccoli, L. \& T. Cabrera. 1994a. Estudio de la hidrografía y el fitoplancton en la isla de Cubagua, Venezuela. Memorias III Congreso de Ciencias del Mar. Marcaba, 45-56 pp.

Troccoli, L. \& T. Cabrera. 1994b. Variaciones de la estructura comunitaria del fitoplancton de la isla de Cubagua. Memorias I Congreso Venezolano de Ciencias Acuáticas, 12-35 pp.

Trujillo, E. \& G. Martínez. 2003. Estimación de la profundidad de trabajo de anzuelos de un palangre atunero prototipo de la flota de altura de isla de Margarita (Venezuela). Invest. Mar., Valparaíso, 31(2): 25-34.

Voinikanis-Mirskii, V.N. 1966. Ejercicios y cálculo para las pesquerías industriales. Industria Alimenticia, Moscú, 366 pp. (en ruso).

Voinikanis-Mirskii, V.N. 1971. Tecnología de la construcción de artes de pesca industrial. Industria Alimenticia, Moscú, 272 pp. (en ruso).

Voinikanis-Mirskii, V.N. 1979. Teoría y diseño de artes de pesca industrial. Material didáctico. Universidad Técnica de la Industria y Economía Pesquera de Astrakhan. Ministerio de Pesquería URSS. Cátedra de Pesca Industrial, 294 pp. (en ruso).

Wiegel, R. 1964. Mooring. In: Oceanographical Engineering. Practice-Hall International Series: pp. 506-509. 